Annales Academiæ Scientiarum Fennicæ

Mathematica

Volumen 38, 2013, 677-689

\title{
BEURLING'S CRITERION AND EXTREMAL METRICS FOR FUGLEDE MODULUS
}

\author{
Matthew Badger \\ Stony Brook University, Department of Mathematics \\ Stony Brook, NY 11794-3651, U.S.A.; badger@math.sunysb.edu
}

\begin{abstract}
For each $1 \leq p<\infty$, we formulate a necessary and sufficient condition for an admissible metric to be extremal for the Fuglede $p$-modulus of a system of measures. When $p=2$, this characterization generalizes Beurling's criterion, a sufficient condition for an admissible metric to be extremal for the extremal length of a planar curve family. In addition, we prove that every Borel function $\varphi: \mathbf{R}^{n} \rightarrow[0, \infty]$ satisfying $0<\int \varphi^{p}<\infty$ is extremal for the $p$-modulus of some curve family in $\mathbf{R}^{n}$.
\end{abstract}

\section{Introduction}

In this note we take a close look at extremal metrics for systems of measures and families of curves. Let us start by recalling Fuglede's definition of modulus [6]. Fix once and for all a measure space $(X, \mathcal{M}, m)$. A collection of measures $\mathbf{E}$ is a measure system (over $\mathcal{M}$ ) if each measure $\mu \in \mathbf{E}$ is defined on the $\sigma$-algebra $\mathcal{M}$. A Borel function $\varphi: X \rightarrow[0, \infty]$ is called a metric and is said to be admissible for $\mathbf{E}$ if $\int \varphi d \mu \geq 1$ for all $\mu \in \mathbf{E}$. (We do not identify two metrics which agree $m$-a.e.) For each $0<p<\infty$, the $p$-modulus of $\mathbf{E}$ is given by

$$
\bmod _{p} \mathbf{E}=\inf \left\{\int \varphi^{p} d m: \varphi \text { is admissible for } \mathbf{E}\right\}
$$

where $\bmod _{p} \mathbf{E}=\infty$ if admissible metrics for $\mathbf{E}$ do not exist.

Example 1. To pick a concrete setting, take $(X, \mathcal{M}, m)=\left(\mathbf{R}^{n}, \mathcal{B}_{n}, m_{n}\right)$ where $m_{n}$ is the Lebesgue measure on the Borel subsets $\mathcal{B}_{n}$ of $\mathbf{R}^{n}$. A (locally rectifiable) curve $\gamma$ in $\mathbf{R}^{n}$ is a concatenation (disjoint union) of countably many images of oneto-one Lipschitz maps $\gamma_{i}:\left[a_{i}, b_{i}\right] \rightarrow \mathbf{R}^{n}$. Each image $\gamma_{i}\left(\left[a_{i}, b_{i}\right]\right)$ is called a piece of $\gamma$; curves may have disjoint or overlapping pieces. (For an alternative definition of a curve, see [17].) The trace of a curve $\gamma$ is the set $\bigcup_{i} \gamma_{i}\left(\left[a_{i}, b_{i}\right]\right)$, i.e. the union of the pieces of $\gamma$. For every curve $\gamma$ in $\mathbf{R}^{n}$ there is a Borel measure $\tilde{\gamma}$ on $\mathbf{R}^{n}$ such that the line integral

$$
\int_{\gamma} f d s=\sum_{i} \int_{a_{i}}^{b_{i}} f\left(\gamma_{i}(t)\right)\left|\gamma_{i}^{\prime}(t)\right| d t
$$

is given by integration against $\tilde{\gamma}$, i.e. $\int_{\gamma} f d s=\int_{\mathbf{R}^{n}} f d \tilde{\gamma}$ for every Borel function $f$. (By the area formula $\tilde{\gamma}=\sum_{i} \tilde{\gamma}_{i}$ where $\tilde{\gamma}_{i}=H^{1}\left\llcorner\gamma_{i}\left(\left[a_{i}, b_{i}\right]\right)\right.$ is the 1-dimensional Hausdorff measure restricted to the set $\gamma_{i}\left(\left[a_{i}, b_{i}\right]\right)$, e.g. see [5].) For all $1 \leq p<\infty$, the $p$-modulus of a family of curves $\Gamma$ in $\mathbf{R}^{n}$ is defined in terms of Fuglede modulus by

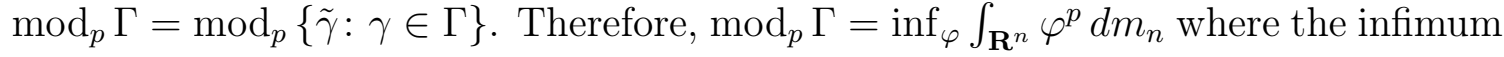

doi:10.5186/aasfm.2013.3826

2010 Mathematics Subject Classification: Primary 31B15; Secondary 28A33, 49K27.

Key words: Beurling's criterion, extremal metric, modulus, extremal length.

The author was partially supported by an NSF postdoctoral fellowship, grant DMS-1203497. 
runs over all Borel functions $\varphi \geq 0$ such that $\int_{\gamma} \varphi d s \geq 1$ for all $\gamma \in \Gamma$. (One could similarly define $\bmod _{p} \Gamma$ for $0<p<1$, but this quantity is always zero.) In the plane, the extremal length $\lambda(\Gamma)=1 / \bmod _{2} \Gamma$ of a curve family $\Gamma$ in $\mathbf{R}^{2}$ is often used instead of its modulus.

An atom in a $\sigma$-algebra $\mathcal{M}$ is a nonempty set $A \in \mathcal{M}$ with the property $B \subsetneq A$, $B \in \mathcal{M} \Rightarrow B=\emptyset$. That is, the only proper measurable subset of an atom is the empty set. If $\varphi: X \rightarrow[0, \infty]$ is a Borel function on $(X, \mathcal{M})$, then $\varphi$ is constant on each atom of $\mathcal{M}$. Given an atom $A \in \mathcal{M}$, the atomic measure $\delta_{A}$ is defined by the rule $\delta_{A}(S)=1$ if $A \subset S$ and $\delta_{A}(S)=0$ otherwise; $\int \varphi d \delta_{A}=\varphi(A)$ for all $\varphi$ and $A$.

Example 2. Let $\mathcal{K}=\left\{K_{1}, \ldots, K_{\ell}\right\}$ be a finite set of pairwise disjoint compact subsets of the Riemann sphere $\hat{\mathbf{C}}$, and let $\Omega \subset \hat{\mathbf{C}}$ be an open set. The transboundary measure space $\left(\hat{\mathbf{C}}, \mathcal{M}_{\mathcal{K}}, m_{\Omega, \mathcal{K}}\right)$ is defined as follows. Let $\mathcal{B}(\hat{\mathbf{C}} \backslash K)$ denote the Borel $\sigma$-algebra on the complement of $K=\bigcup_{i=1}^{\ell} K_{i}$. Then $\mathcal{M}_{\mathcal{K}}$ is the smallest $\sigma$-algebra generated by $\mathcal{B}(\hat{\mathbf{C}} \backslash K) \cup \mathcal{K}$. The atoms of $\mathcal{M}_{\mathcal{K}}$ are the singletons $\{x\}$ with $x \in \hat{\mathbf{C}} \backslash K$ and the sets $K_{1}, \ldots, K_{\ell}$. We define the measure $m_{\Omega, \mathcal{K}}=H^{2}\left\llcorner(\Omega \backslash K)+\sum_{i=1}^{\ell} \delta_{K_{i}}\right.$ where $H^{2}\llcorner(\Omega \backslash K)$ is 2-dimensional Hausdorff measure on $\Omega \backslash K$. Let $\gamma:[a, b] \rightarrow \hat{\mathbf{C}}$ be a one-to-one continuous map, let $\operatorname{Im} \gamma=\gamma([a, b])$ be its image, and assume that $\operatorname{Im} \gamma \cap(\Omega \backslash K)$ is locally rectifiable. Then we define a measure $\hat{\gamma}$ on $\left(\hat{\mathbf{C}}, \mathcal{M}_{\mathcal{K}}\right)$ by

$$
\hat{\gamma}=H^{1}\left\llcorner\operatorname{Im} \gamma \cap(\Omega \backslash K)+\sum_{i: \operatorname{Im} \gamma \cap K_{i} \neq \emptyset} \delta_{K_{i}}\right.
$$

Suppose that $(X, \mathcal{M}, m)=\left(\hat{\mathbf{C}}, \mathcal{M}_{\mathcal{K}}, m_{\Omega, \mathcal{K}}\right)$. The transboundary modulus $\bmod _{\Omega, \mathcal{K}} \Gamma$ of a collection $\Gamma$ of one-to-one continuous maps $\gamma:[a, b] \rightarrow \hat{\mathbf{C}}$ is defined via Fuglede modulus by $\bmod _{\Omega, \mathcal{K}} \Gamma=\bmod _{2}\{\hat{\gamma}: \gamma \in \Gamma$ and $\operatorname{Im} \gamma \cap(\Omega \backslash K)$ is locally rectifiable $\}$. Thus, an admissible metric $\varphi: \hat{\mathbf{C}} \rightarrow[0, \infty]$ satisfies

$$
\int_{\operatorname{Im} \gamma \cap(\Omega \backslash K)} \varphi d s+\sum_{i: \operatorname{Im} \gamma \cap K_{i} \neq \emptyset} \varphi\left(K_{i}\right) \geq 1
$$

for every $\gamma \in \Gamma$ such that $\operatorname{Im} \gamma \cap(\Omega \backslash K)$ is locally rectifiable, and

$$
\bmod _{\Omega, \mathcal{K}} \Gamma=\inf _{\varphi} \int_{\Omega \backslash K} \varphi^{2} d H^{2}+\sum_{i=1}^{\ell} \varphi\left(K_{i}\right)^{2}
$$

where the infimum runs over all admissible metrics $\varphi: \hat{\mathbf{C}} \rightarrow[0, \infty]$. The reciprocal $\lambda_{\Omega, \mathcal{K}}(\Gamma)=1 / \bmod _{\Omega, \mathcal{K}} \Gamma$ of transboundary modulus is transboundary extremal length.

The definition of extremal length is due to Beurling and has roots in the classical length-area principle for conformal maps; see Jenkins [12] for a historical overview. Since the introduction of extremal length by Ahlfors and Beurling [2], the modulus of a curve family has become a widely-used tool, employed in geometric function theory [1, 7, 15], quasiconformal and quasiregular mappings [17, 18], dynamical systems $[8,13]$, and analysis on metric spaces $[10,11]$. The transboundary extremal length of a curve family was introduced by Schramm [16] to study uniformization on countablyconnected domains. Recently Bonk [4] used transboundary modulus in a crucial way to obtain uniformization results on Sierpiński carpets in the plane. For applications of modulus of measures, see Fuglede's original applications in [6], Hakobyan's work 
on the conformal dimension of sets [9], and Bishop and Hakobyan's recent paper on the frequency of dimension distortion by quasisymmetric maps [3].

A few nice properties of modulus are apparent from the definition. First if $\mathbf{E} \subset \mathbf{F}$ then $\bmod _{p} \mathbf{E} \leq \bmod _{p} \mathbf{F}$. Second $\bmod _{p} \bigcup_{i=1}^{\infty} \mathbf{E}_{i} \leq \sum_{i=1}^{\infty} \bmod _{p} \mathbf{E}_{i}$ for any sequence of measure systems. Since $\bmod _{p} \emptyset=0$, this says that modulus is an outer measure on measure systems. A third useful property is that every admissible metric gives an upper bound on modulus, i.e. $\bmod _{p} \mathbf{E} \leq \int \varphi^{p} d m$ for all admissible metrics $\varphi$.

If the infimum in the definition of the modulus of a measure system $\mathbf{E}$ is obtained by an admissible metric $\varphi$, i.e. if $\bmod _{p} \mathbf{E}=\int \varphi^{p} d m$, then the metric $\varphi$ is said to be extremal for the $p$-modulus of $\mathbf{E}$. Naturally one may ask whether an extremal metric always exists, and if so, to what extent is an extremal metric uniquely determined. Unfortunately simple examples (see Example 5 below) show that the existence and uniqueness of extremal metrics fails for general measure systems. Nevertheless, Fuglede [6] proved that when $1<p<\infty$, a measure system always admits an extremal metric, after removing an exceptional system of measures.

Fuglede's Lemma. Let $1<p<\infty$. Let $\mathbf{E}$ be a measure system. If $\bmod _{p} \mathbf{E}<$ $\infty$, then there exists a measure system $\mathbf{N} \subset \mathbf{E}$ such that $\bmod _{p} \mathbf{N}=0$ and $\mathbf{E} \backslash \mathbf{N}$ admits an extremal metric $\varphi$.

The uniqueness of an extremal metric for the $p$-modulus of a measure system also holds when $1<p<\infty$, up to redefinition of the metric on a set of $m$-measure zero. This can be seen as follows. Suppose that $\varphi, \psi \in L^{p}(m)$ are two extremal metrics for the $p$-modulus of a measure system $\mathbf{E}$. Then the averaged metric $\chi=\frac{1}{2} \varphi+\frac{1}{2} \psi$ is still admissible for $\mathbf{E}$ and $\left(\bmod _{p} \mathbf{E}\right)^{1 / p} \leq\|\chi\|_{p} \leq \frac{1}{2}\|\varphi\|_{p}+\frac{1}{2}\|\psi\|_{p}=\left(\bmod _{p} \mathbf{E}\right)^{1 / p}$. Thus, $\left\|\frac{1}{2} \varphi+\frac{1}{2} \psi\right\|_{p}=\frac{1}{2}\|\varphi\|_{p}+\frac{1}{2}\|\psi\|_{p}$. By the condition for equality in Minkowski's inequality and the assumption that $\|\varphi\|_{p}=\|\psi\|_{p}<\infty$, one obtains $\varphi=\psi m$-a.e., as desired.

A fundamental problem working with modulus is to identify an extremal metric for a given measure system or curve family if one exists. Beurling found a general sufficient condition which guarantees that an admissible metric for a curve family in the plane is extremal for its extremal length.

Beurling's Criterion. [1, Theorem 4.4] Let $\Gamma$ be a curve family in $\mathbf{R}^{2}$ and let $\varphi$ be an admissible metric for $\Gamma$ such that $0<\int_{\mathbf{R}^{2}} \varphi^{2}<\infty$. Suppose that there exists a curve family $\Gamma_{0}$ in $\mathbf{R}^{2}$ such that

(1) $\Gamma_{0} \subset \Gamma$,

(2) $\int_{\gamma} \varphi d s=1$ for every $\gamma \in \Gamma_{0}$, and

(3) for all $f \in L^{2}\left(\mathbf{R}^{2}\right)$ taking values in $[-\infty, \infty]$ : if $\int_{\gamma} f d s \geq 0$ for all $\gamma \in \Gamma_{0}$, then $\int_{\mathbf{R}^{2}} f \varphi \geq 0$.

Then $\varphi$ is an extremal metric for the extremal length of $\Gamma$, i.e. $\lambda(\Gamma)=\left(\int_{\mathbf{R}^{2}} \varphi^{2}\right)^{-1}$.

Let us see Beurling's criterion in action, in a standard example.

Example 3. Let $R$ be a rectangle with side lengths $a \leq b$. Let $\Gamma$ be the family of all curves in $R$ with connected trace which join opposite edges in $R$ (see Figure 1). We claim that $\varphi=\frac{1}{a} \chi_{R}$ is an extremal metric for $\Gamma$, and thus,

$$
\lambda(\Gamma)=\left(\int_{R} \frac{1}{a^{2}}\right)^{-1}=a / b .
$$


First $\varphi$ is admissible for $\Gamma$, because every curve connecting opposite edges in $R$ travels at least Euclidean distance $a$ (the distance between the edges of length $b$ ). Beurling's criterion holds with $\Gamma_{0}=\{\gamma(t): t \in[0, b]\}$ equal to the family of straight line segments connecting (and orthogonal to) a pair of opposite sides of length $b$. Conditions (1) and (2) hold by definition. And (3) follows from Fubini's theorem: if $\int_{\gamma(t)} f d s \geq 0$ for all $\gamma(t) \in \Gamma_{0}$, then $\int_{\mathbf{R}^{2}} f \varphi=\frac{1}{a} \int_{R} f=\frac{1}{a} \int_{0}^{b} \int_{\gamma(t)} f d s d t \geq 0$. Therefore, $\varphi$ is extremal for $\lambda(\Gamma)$.

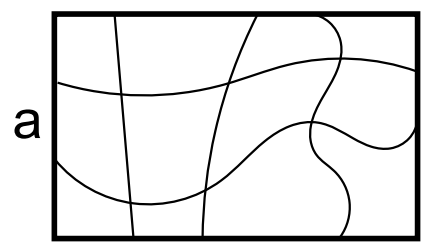

b

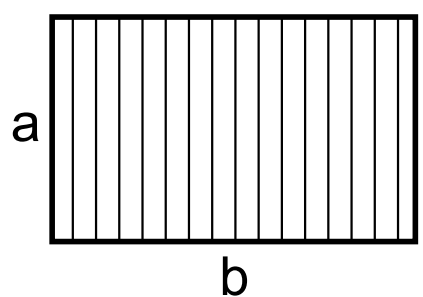

Figure 1. Curve families $\Gamma$ and $\Gamma_{0}$ in Examples 3 and 4.

The converse to Beurling's criterion fails for the simple reason that $\Gamma$ may not contain any curves $\gamma$ such that $\int_{\gamma} \varphi d s=1$.

Example 4. Once again let $R$ be a rectangle with side lengths $a \leq b$, and let $\Gamma$ and $\Gamma_{0}$ be the curve families from Example 3. We claim that $\varphi=\frac{1}{a} \chi_{R}$ is an extremal metric for $\Gamma_{*}=\Gamma \backslash \Gamma_{0}$. However, since $\Gamma_{*}$ does not contain any curves $\gamma$ such that $\int_{\gamma} \varphi d s=1$, we cannot use Beurling's criterion to show that $\varphi$ is extremal for $\Gamma_{*}$. Let $\psi$ be an admissible metric for $\Gamma_{*}$. Fix $\gamma(t) \in \Gamma_{0}$. Then one can find a sequence of curves $\gamma^{k}(t) \in \Gamma_{*}$ such that $\int_{\gamma^{k}(t)} \psi d s \rightarrow \int_{\gamma(t)} \psi d s$. (For example, if $\gamma(t)=[0, a]$, then take $\gamma^{k}(t)=[1 / k, 0] \sqcup[0, a]$ where $\sqcup$ denotes concatenation.) In particular, it follows that $\int_{\gamma(t)} \psi d s \geq 1$ for all $\gamma(t) \in \Gamma_{0}$. Integrating across all $\gamma(t) \in \Gamma_{0}$, invoking Fubini's theorem and applying the Cauchy-Schwarz inequality gives

$$
b \leq \int_{0}^{b} \int_{\gamma(t)} \psi d s d t=\int_{R} \psi \leq\left(\int_{R} \psi^{2}\right)^{1 / 2}(a b)^{1 / 2} \leq\left(\int_{\mathbf{R}^{2}} \psi^{2}\right)^{1 / 2}(a b)^{1 / 2} .
$$

Thus, $\left(\int_{\mathbf{R}^{2}} \psi^{2}\right)^{-1} \leq a / b$ for every metric $\psi$ that is admissible for $\Gamma_{*}$. Since this upper bound is obtained by $\varphi$, we conclude that $\varphi$ is extremal for $\lambda\left(\Gamma_{*}\right)$.

A partial converse to Beurling's criterion is presented in Ohtsuka [14, §2.3] in the special case $\Gamma=\Gamma_{0}$ : if $\varphi$ is extremal for $\Gamma$, then (3) holds for all $f \in L^{2}\left(\mathbf{R}^{2}\right)$. Wolf and Zwiebach [19, p. 38] have also established "a partial local converse to Beurling's criterion" for certain classes of metrics on Riemann surfaces.

\section{Statement of results}

The main goal of this note is to show that Beurling's criterion can be modified to become a necessary and sufficient test for extremal metrics. In fact, we establish a characterization of extremal metrics in the general setting of Fuglede modulus, when $1<p<\infty$ and when $p=1$.

Theorem 1. (Extremal metrics in $L^{p}$ ) Let $1<p<\infty$. Let $\mathbf{E}$ be a measure system and let $\varphi$ be an admissible metric for $\mathbf{E}$ such that $\varphi \in L^{p}(m)$. Then $\varphi$ is extremal for the p-modulus of $\mathbf{E}$ if and only if

$\left(B_{p}\right)$ there exists a measure system $\mathbf{F}$ such that 
(a) $\bmod _{p} \mathbf{E} \cup \mathbf{F}=\bmod _{p} \mathbf{E}$,

(b) $\int \varphi d \nu=1$ for every $\nu \in \mathbf{F}$, and

(c) for all $f \in L^{p}(m)$ taking values in $[-\infty, \infty]$ : if $\int f d \nu \geq 0$ for all $\nu \in \mathbf{F}$, then $\int f \varphi^{p-1} d m \geq 0$.

Theorem 2. (Extremal metrics in $L^{1}$ ) Let $\mathbf{E}$ be a measure system and let $\varphi$ be an admissible metric for $\mathbf{E}$ such that $\varphi \in L^{1}(m)$. Then $\varphi$ is extremal for the 1-modulus of $\mathbf{E}$ if and only if

$\left(B_{1}\right)$ there exists a measure system $\mathbf{F}$ such that

(a) $\bmod _{1} \mathbf{E} \cup \mathbf{F}=\bmod _{1} \mathbf{E}$,

(b) $\int \varphi d \nu=1$ for every $\nu \in \mathbf{F}$, and

(c) for all $f \in L^{1}(m)$ taking values in $[-\infty, \infty]$ such that $\varphi(x)=0$ implies $f(x) \geq 0$ : if $\int f d \nu \geq 0$ for all $\nu \in \mathbf{F}$, then $\int f d m \geq 0$.

Remark 1. We label the conditions $\left(B_{p}\right)$ in Theorems 1 and 2 in honor of Beurling. When $p=2$ and $\mathbf{F} \subset \mathbf{E}$, (a) holds vacuously and $\left(B_{2}\right)$ is Beurling's criterion.

Remark 2. In Theorems 1 and 2, if $\varphi$ is extremal for $\bmod _{p} \mathbf{E}$, then there exists $\mathbf{F}$ satisfying $\left(B_{p}\right)$ such that for every $\nu \in \mathbf{F}$ there exist $\mu \in \mathbf{E}$ and $0<c \leq 1$ such that $\nu=c \mu$.

Remark 3. In Theorems 1 and 2 the case $\mathbf{F}=\emptyset$ is allowed. Note condition $\left(B_{p}\right)$ holds with $\mathbf{F}=\emptyset$ if and only if $\varphi=0 \mathrm{~m}$-almost everywhere.

The proofs of Theorems 1 and 2 will be given in sections 3 and 4, respectively. (A curious reader may jump to the proofs immediately.) We now demonstrate the use of the theorems in a simple, yet enlightening example, which shows the varied behavior of the $p$-modulus for different values of $p$.

Example 5. Assume that $A \in \mathcal{M}$ and $0<m(A)<\infty$. Let $\mathbf{E}_{A}=\{m\llcorner A\}$ where $m\llcorner A$ denotes the measure $m$ restricted to the set $A$. Then

$$
\bmod _{p} \mathbf{E}_{A}= \begin{cases}\inf \left\{m(B)^{1-p}: B \subset A, m(B)>0\right\}, & \text { if } 0<p \leq 1, \\ m(A)^{1-p}, & \text { if } 1 \leq p<\infty .\end{cases}
$$

This will be checked in four steps.

Let $1<p<\infty$. We will show that $\varphi_{A}=m(A)^{-1} \chi_{A}$ is extremal for $\bmod _{p} \mathbf{E}_{A}$, and hence, $\bmod _{p} \mathbf{E}_{A}=\int_{A} m(A)^{-p} d m=m(A)^{1-p}$. Clearly $\varphi_{A} \in L^{p}(m)$ and $\varphi_{A}$ is admissible for $\mathbf{E}_{A}$. Let us check that $\left(B_{p}\right)$ holds with $\mathbf{F}=\mathbf{E}_{A}$. Conditions (a) and (b) hold immediately. For condition (c), $\int f \varphi_{A}^{p-1} d m=m(A)^{1-p} \int_{A} f d m \geq 0$ whenever $\int f d\left(m\llcorner A) \geq 0\right.$. Thus, $\varphi_{A}$ is extremal for $\bmod _{p} \mathbf{E}_{A}$, by Theorem 1 .

The case $p=1$ is similar, except that there is no longer a unique extremal metric. Let $B \subset A$ be any subset such that $m(B)>0$ and let $\varphi_{B}=m(B)^{-1} \chi_{B}$. Then $\varphi_{B} \in L^{1}(m)$ and $\varphi_{B}$ is admissible for $\mathbf{E}_{A}$. We will check that $\left(B_{1}\right)$ holds with $\mathbf{F}=\mathbf{E}_{A}$. Conditions (a) and (b) are immediate. To verify condition (c) of $\left(B_{1}\right)$, assume that $f \in L^{1}(m)$ takes values in $[-\infty, \infty], f(x) \geq 0$ whenever $\varphi_{B}(x)=0$ and $\int f d\left(m\llcorner A) \geq 0\right.$. Then $\int f d m=\int_{A^{c}} f d m+\int_{A} f d m \geq 0$, where the first term is non-negative since $\varphi_{B}(x)=0$ on $A^{c}$. Thus, $\varphi_{B}$ is extremal for $\bmod _{1} \mathbf{E}_{A}$, by Theorem 2 , so that $\bmod _{1} \mathbf{E}_{A}=\int \varphi_{B} d m=1$ for every $B \subset A$ with $m(B)>0$.

Next let $0<p<1$ and suppose that $A$ has subsets of arbitrarily small positive measure. Then we can find a sequence of subsets $B_{k} \subset A$ with $m\left(B_{k}\right)>0$ such 
that $\lim _{k \rightarrow \infty} m\left(B_{k}\right)=0$. The normalized characteristic functions $\varphi_{k}=m\left(B_{k}\right)^{-1} \chi_{B_{k}}$ are admissible for $\mathbf{E}_{A}$. Hence $\bmod _{p} \mathbf{E}_{A} \leq \int \varphi_{k}^{p} d m=m\left(B_{k}\right)^{1-p} \rightarrow 0$ as $k \rightarrow \infty$, since $0<p<1$. Therefore, $\bmod _{p} \mathbf{E}_{A}=0=\inf \left\{m(B)^{1-p}: B \subset A, m(B)>0\right\}$. However, there is no extremal metric for $\bmod _{p} \mathbf{E}_{A}$, because no function $\psi \geq 0$ satisfies $\int \psi d\left(m\llcorner A) \geq 1\right.$ and $\int \psi^{p} d m=0$ simultaneously.

Finally, let $0<p<1$, but suppose that $A$ does not possess subsets of arbitrarily small positive measure. Then $m\left\llcorner A=c_{1} \delta_{A_{1}}+\cdots+c_{k} \delta_{A_{k}}\right.$ is a linear combination of atomic measures, where each atom $A_{i} \in \mathcal{M}$ and $A_{i} \cap A_{j}=\emptyset$ whenever $i \neq j$. By relabeling, we may assume that $0<c_{1} \leq c_{j}$ for all $2 \leq j \leq k$. Let $\rho \geq 0$ be an admissible metric for $\mathbf{E}_{A}$ such that $\int \rho d(m\llcorner A)=1$. (Here we can ask for equality, because $\mathbf{E}_{A}$ consists of one element.) Define $\eta_{j}=\rho\left(A_{j}\right) c_{j}$ for all $j$. Then $\sum_{j=1}^{k} \eta_{j}=\sum_{j=1}^{k}\left(\eta_{j} / c_{j}\right) c_{j}=\int \rho d\left(m\llcorner A)=1\right.$. Thus, $0 \leq \eta_{j} \leq 1$ for all $j$, and

$$
\int \rho^{p} d m \geq \sum_{j=1}^{k}\left(\eta_{j} / c_{j}\right)^{p} c_{j}=\sum_{j=1}^{k} \eta_{j}^{p} c_{j}^{1-p} \geq \sum_{j=1}^{k} \eta_{j} c_{1}^{1-p}=c_{1}^{1-p}
$$

Since the lower bound $\int \rho^{p} d m \geq c_{1}^{1-p}$ is obtained by the metric $\rho=m\left(A_{1}\right)^{-1} \chi_{A_{1}}$, we conclude that $\bmod _{p} \mathbf{E}_{A}=m\left(A_{1}\right)^{1-p}=\inf \left\{m(B)^{1-p}: B \subset A, m(B)>0\right\}$.

Remark 4. With the same notation as in the previous example, $\varphi_{A}=m(A)^{-1} \chi_{A}$ also satisfies condition $\left(B_{p}\right)$ with $\mathbf{F}=\mathbf{E}_{A}$ for $0<p<1$. But $\bmod _{p} \mathbf{E}_{A} \neq \int \varphi_{A}^{p} d m$ when $0<p<1$ unless $m\left\llcorner A=c \delta_{A}\right.$. Thus, Example 5 shows that condition $\left(B_{p}\right)$ from Theorem 1 is not a sufficient test for extremal metrics when $0<p<1$.

The characterizations of extremal metrics for the $p$-modulus of measure systems in Theorems 1 and 2 also hold for curve families in $\mathbf{R}^{n}$. In particular, assuming that an extremal metric for $\mathbf{E}=\{\tilde{\gamma}: \gamma \in \Gamma\}$ exists, one can find a measure system $\mathbf{F}$ satisfying condition $\left(B_{p}\right)$, where $\mathbf{F}$ is also associated to a family of curves in $\mathbf{R}^{n}$.

Corollary 1. (Extremal metrics in $L^{p}$ for curves) Let $1<p<\infty$. Let $\Gamma$ be a curve family in $\mathbf{R}^{n}$ and let $\varphi$ be an admissible metric for $\Gamma$ such that $\varphi \in L^{p}\left(\mathbf{R}^{n}\right)$. Then $\varphi$ is extremal for the $p$-modulus of $\Gamma$ if and only if

$\left(B_{p}^{\prime}\right)$ there exists a curve family $\Gamma^{\prime}$ in $\mathbf{R}^{n}$ such that

(a) $\bmod _{p} \Gamma \cup \Gamma^{\prime}=\bmod _{p} \Gamma$,

(b) $\int_{\gamma} \varphi d s=1$ for every $\gamma \in \Gamma^{\prime}$, and

(c) for all $f \in L^{p}\left(\mathbf{R}^{n}\right)$ taking values in $[-\infty, \infty]$ : if $\int_{\gamma} f d s \geq 0$ for all $\gamma \in \Gamma^{\prime}$, then $\int_{\mathbf{R}^{n}} f \varphi^{p-1} \geq 0$.

Corollary 2. (Extremal metrics in $L^{1}$ for curves) Let $\Gamma$ be a curve family in $\mathbf{R}^{n}$ and let $\varphi$ be an admissible metric for $\Gamma$ such that $\varphi \in L^{1}\left(\mathbf{R}^{n}\right)$. Then $\varphi$ is extremal for the 1-modulus of $\Gamma$ if and only if

$\left(B_{1}^{\prime}\right)$ there exists a curve family $\Gamma^{\prime}$ in $\mathbf{R}^{n}$ such that

(a) $\bmod _{1} \Gamma \cup \Gamma^{\prime}=\bmod _{1} \Gamma$,

(b) $\int_{\gamma} \varphi d s=1$ for every $\gamma \in \Gamma^{\prime}$, and

(c) for all $f \in L^{1}\left(\mathbf{R}^{n}\right)$ taking values in $[-\infty, \infty]$ such that $\varphi(x)=0$ implies $f(x) \geq 0$ : if $\int_{\gamma} f d s \geq 0$ for all $\gamma \in \Gamma^{\prime}$, then $\int_{\mathbf{R}^{n}} f \geq 0$.

Remark 5. In Corollaries 1 and 2 , if $\varphi$ is extremal for $\bmod _{p} \Gamma$, then there exists $\Gamma^{\prime}$ satisfying $\left(B_{p}^{\prime}\right)$ such that every curve $\gamma^{\prime} \in \Gamma^{\prime}$ is a subcurve of some curve $\gamma \in \Gamma$. 
Remark 6. In Corollaries 1 and 2 the case $\Gamma^{\prime}=\emptyset$ is allowed. Note condition $\left(B_{p}^{\prime}\right)$ holds with $\Gamma^{\prime}=\emptyset$ if and only if $\varphi=0$ Lebesgue almost everywhere.

The auxiliary curve family $\Gamma^{\prime}$ that is required to test condition $\left(B_{p}^{\prime}\right)$ is not unique. In the next example, we exhibit disjoint curve families $\Gamma_{0}$ and $\Gamma_{1}$ such that condition $\left(B_{2}^{\prime}\right)$ holds with the auxiliary curve family $\Gamma^{\prime}=\Gamma_{i}, i=0,1$.

Example 6. Let $R$ be a rectangle with side lengths $a \leq b$, and let $\Gamma$ and $\Gamma_{0}$ be the curve families from Example 3. Above we showed that condition $\left(B_{2}^{\prime}\right)$ (i.e. Beurling's criterion) holds for $\Gamma$ and $\varphi=\frac{1}{a} \chi_{R}$ using the auxiliary curve family $\Gamma^{\prime}=\Gamma_{0} \subset \Gamma$. Thus, $\bmod _{2} \Gamma=\int_{R}(1 / a)^{2}=b / a$ by Corollary 1 . Alternatively let $\Gamma_{1}$ be the curve family described as follows (see Figure 2). For each $\gamma(t) \in \Gamma_{0}$, there correspond exactly two curves $\gamma^{\prime}(t)$ and $\gamma^{\prime \prime}(t)$ in $\Gamma_{1}$. If the curve $\gamma(t)=[P, Q]$, then the curves $\gamma^{\prime}(t)$ and $\gamma^{\prime \prime}(t)$ are given by

$$
\gamma^{\prime}(t)=\left[P, \frac{P+Q}{2}\right] \sqcup\left[\frac{P+Q}{2}, P\right] \quad \text { and } \quad \gamma^{\prime \prime}(t)=\left[Q, \frac{P+Q}{2}\right] \sqcup\left[\frac{P+Q}{2}, Q\right]
$$

where $\sqcup$ denotes concatenation.
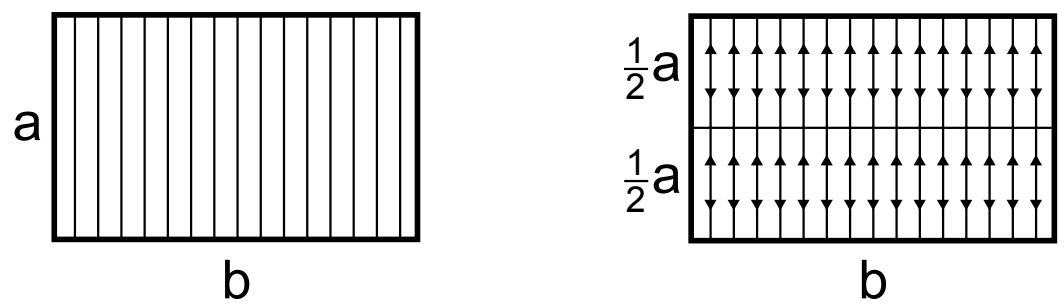

Figure 2. Curve families $\Gamma_{0}$ and $\Gamma_{1}$ in Example 6 .

In other words, each curve in $\Gamma_{1}$ travels along a straight path starting at and perpendicular to an edge of side length $b$; half-way across to the other side, the curve reverses direction and returns to its starting point. We now check that $\left(B_{2}^{\prime}\right)$ holds for $\Gamma$ and $\varphi$ with $\Gamma^{\prime}=\Gamma_{1}$. A quick computation shows that $\int_{\gamma} \varphi d s=1$ for all $\gamma \in \Gamma_{1}$. Hence condition (b) holds. For (a), we have $\bmod _{2} \Gamma \leq \bmod _{2} \Gamma \cup \Gamma_{1} \leq$ $\int_{\mathbf{R}^{2}} \varphi^{2}=\bmod _{2} \Gamma$, since $\varphi$ is admissible for $\Gamma \cup \Gamma_{1}$ and since (we already know that) $\varphi$ is extremal for $\Gamma$. It remains to check (c). If $f \in L^{2}\left(\mathbf{R}^{2}\right)$ and $\int_{\gamma} f d s \geq 0$ for all $\gamma \in \Gamma_{1}$, then

$$
\begin{aligned}
2 \int_{\mathbf{R}^{2}} f \varphi & =\frac{2}{a} \int_{R} f=\frac{2}{a} \int_{0}^{b} \int_{\gamma(t)} f d s d t=\frac{1}{a} \int_{0}^{b} \int_{\gamma(t) \sqcup \gamma(t)} f d s d t \\
& =\frac{1}{a} \int_{0}^{b} \int_{\gamma^{\prime}(t) \sqcup \gamma^{\prime \prime}(t)} f d s d t=\frac{1}{a} \int_{0}^{b} \int_{\gamma^{\prime}(t)} f d s d t+\frac{1}{a} \int_{0}^{b} \int_{\gamma^{\prime \prime}(t)} f d s d t \geq 0 .
\end{aligned}
$$

Thus, condition (c) holds too, and we have reached the end of the example.

It is of course possible to specialize Theorems 1 and 2 to other settings. For instance, a reader familiar with analysis on metric spaces will have no difficulty adapting Corollaries 1 and 2 to the metric space setting. In [4, §11], Bonk notes that Beurling's criterion can be adapted to produce a sufficient test for extremal metrics for the transboundary modulus of a curve family in the Riemann sphere. Using Theorem 1 and the proof of Corollary 1, one can also formulate a necessary and sufficient test for extremal metrics for transboundary modulus. 
So far we have found a characterization of extremal metrics for the $p$-modulus of a measure system or curve family when $1 \leq p<\infty$. A related problem is to identify those metrics which are extremal for the $p$-modulus of some measure system or curve family. The next result gives a solution to this problem for measure systems.

Theorem 3. If $\varphi: X \rightarrow[0, \infty]$ is a metric and $\varphi<\infty m$-a.e., then $\varphi$ is extremal for the p-modulus of $\mathbf{E}_{\varphi}=\left\{\mu\right.$ defined on $\left.\mathcal{M}: \int \varphi d \mu \geq 1\right\}$ for all $0<p<\infty$.

Proof. Let $\mathcal{A}$ be the collection of atoms in $\mathcal{M}$. Note that the scaled atomic measure $\mu_{A}=\varphi(A)^{-1} \delta_{A} \in \mathbf{E}_{\varphi}$ for all $A \in \mathcal{A}$ such that $0<\varphi(A)<\infty$. Let $\psi$ be an admissible metric for $\mathbf{E}_{\varphi}$. Then $\psi(A) / \varphi(A)=\int \psi d \mu_{A} \geq 1$ when $0<\varphi(A)<\infty$. Also, $\psi(A) \geq \varphi(A)$ when $\varphi(A)=0$. Thus, if $\varphi<\infty m$-a.e., then $\psi \geq \varphi m$-a.e., and $\int \psi^{p} d m \geq \int \varphi^{p} d m$ for all $0<p<\infty$. Therefore, $\bmod _{p} \mathbf{E}_{\varphi}=\int \varphi^{p} d m$ for all $0<p<\infty$.

We can establish a similar result for curve families in $\mathbf{R}^{n}$. The basic philosophy, suggested by the proof of Theorem 3, is that one needs to approximate the measures $\delta_{x}$ at points where $\varphi(x)>0$ by sequences of curves. See section 6 for details.

Theorem 4. If $\varphi: \mathbf{R}^{n} \rightarrow[0, \infty]$ is Borel, then $\varphi$ is extremal for the $p$-modulus of $\Gamma_{\varphi}=\left\{\right.$ curve $\gamma$ in $\left.\mathbf{R}^{n}: \int_{\gamma} \varphi d s \geq 1\right\}$ for all $1 \leq p<\infty$ such that $0<\int_{\mathbf{R}^{n}} \varphi^{p}<\infty$.

The plan for the remainder of the note is as follows. In the next two sections, we prove the characterizations of extremal metrics for the $p$-modulus of a measure system from above, in the cases $1<p<\infty$ (section 3 ) and $p=1$ (section 4 ). Then we turn our attention to extremal metrics for families of curves in $\mathbf{R}^{n}$. In section 5 , we show how the proofs of Theorems 1 and 2 must be modified to obtain Corollaries 1 and 2. Finally, we give the proof of Theorem 4 in section 6 .

\section{Proof of Theorem 1 (Extremal metrics in $L^{p}$ )}

Let $1<p<\infty$. Let $\mathbf{E}$ be a measure system and let $\varphi$ be an admissible metric for $\mathbf{E}$ such that $\varphi \in L^{p}(m)$. If $\varphi=0 m$-a.e., then $\varphi$ is extremal for the $p$-modulus of $\mathbf{E}$ and condition $\left(B_{p}\right)$ holds with $\mathbf{F}=\emptyset$. Thus, we assume that $0<\int \varphi^{p} d m<\infty$.

We shall start with the proof that condition $\left(B_{p}\right)$ implies that $\varphi$ is extremal, by mimicking the proof of Beurling's criterion in Ahlfors [1]. Suppose that $\left(B_{p}\right)$ holds for some measure system $\mathbf{F}$ satisfying (a), (b) and (c). Since the metric $\varphi$ is admissible for $\mathbf{E}, \varphi$ is also admissible for $\mathbf{E} \cup \mathbf{F}$, by (b). Let $\psi$ be a competing admissible metric for $\bmod _{p} \mathbf{E} \cup \mathbf{F}$, so that $\int \psi^{p} d m \leq \int \varphi^{p} d m<\infty$. Then $\int \psi d \nu \geq 1=\int \varphi d \nu$ for all $\nu \in \mathbf{F}$, by (b). Hence $f=\psi-\varphi \in L^{p}(m)$ and $\int f d \nu \geq 0$ for all $\nu \in \mathbf{F}$. By (c), we conclude that $\int(\psi-\varphi) \varphi^{p-1} \geq 0$. Then

$$
\int \varphi^{p} d m \leq \int \psi \varphi^{p-1} d m \leq\left(\int \psi^{p} d m\right)^{1 / p}\left(\int \varphi^{p} d m\right)^{(p-1) / p}
$$

where the second inequality is Hölder's inequality. Since $0<\int \varphi^{p} d m<\infty$, we get that $\int \varphi^{p} d m \leq \int \psi^{p} d m$. Thus, $\varphi$ is extremal for the $p$-modulus of $\mathbf{E} \cup \mathbf{F}$. Finally, $\bmod _{p} \mathbf{E} \leq \int \varphi^{p} d m=\bmod _{p} \mathbf{E} \cup \mathbf{F}=\bmod _{p} \mathbf{E}$, by (a). Therefore, $\varphi$ is extremal for the $p$-modulus of $\mathbf{E}$.

For the reverse direction, we require a short lemma. 
Lemma 1. Let $1<p<\infty$. If $\varphi, f \in L^{p}(m)$ take values in $[-\infty, \infty]$ and $\varphi \geq 0$, then

$$
\int\left[(\varphi+\varepsilon f)^{+}\right]^{p} d m=\int_{\{\varphi+\varepsilon f>0\}}\left[\varphi^{p}+p \varepsilon f \varphi^{p-1}\right] d m+o(\varepsilon) \cdot \varepsilon
$$

where $o(\varepsilon) \rightarrow 0$ as $\varepsilon \rightarrow 0$.

Proof. Let $1<p<\infty$ and let $\varphi, f \in L^{p}(m)$ be given. Assume that the functions $\varphi$ and $f$ take values in $[-\infty, \infty]$ and $\varphi \geq 0$. Fix $\varepsilon \neq 0$ and set $P=\{\varphi+\varepsilon f>0\}$. By the mean value theorem, for all $x \in P$ such that $\varphi(x)$ and $f(x)$ are both finite, there exists $\delta=\delta(x)$ between 0 and $\varepsilon$ such that $(\varphi+\varepsilon f)^{p}-\varphi^{p}=\left[p(\varphi+\delta f)^{p-1} f\right] \varepsilon$. In particular, this holds at $m$-a.e. $x \in P$, because $\varphi, f \in L^{p}(m)$, and the function $\delta: P \rightarrow \mathbf{R}$ is measurable, because $\varphi$ and $f$ are measurable. Hence

$$
\int_{P}(\varphi+\varepsilon f)^{p} d m=\int_{P}\left[\varphi^{p}+p \varepsilon f \varphi^{p-1}\right] d m+\varepsilon \int_{P} p f\left[(\varphi+\delta f)^{p-1}-\varphi^{p-1}\right] d m .
$$

The lemma follows, because the second integral in the displayed equation vanishes as $\varepsilon \rightarrow 0$ by the dominated convergence theorem.

Now suppose that $\varphi$ is extremal for the $p$-modulus of $\mathbf{E}$. Break $\mathbf{E}=\mathbf{E}_{0} \cup \mathbf{E}_{\infty}$ into a union of two measure systems where $\mathbf{E}_{0}=\left\{\mu \in \mathbf{E}: 1 \leq \int \varphi d \mu<\infty\right\}$ and $\mathbf{E}_{\infty}=\left\{\mu \in \mathbf{E}: \int \varphi d \mu=\infty\right\}$. Since $\varphi \in L^{p}(m)$, we have $\bmod _{p} \mathbf{E}_{\infty}=0$, because $\varepsilon \varphi$ is admissible for $\mathbf{E}_{\infty}$ for all $\varepsilon>0$. It follows that $\bmod _{p} \mathbf{E}_{0}=\bmod _{p} \mathbf{E}=\int \varphi^{p} d m$; that is, $\varphi$ is extremal for the $p$-modulus of $\mathbf{E}_{0}$, as well. Moreover, $\mathbf{E}_{0}$ is nonempty, since $\bmod _{p} \mathbf{E}_{0}>0$. Recall that we want to show that condition $\left(B_{p}\right)$ holds. Assign $\mathbf{F}$ to be the family of all measures $\nu$ defined on $\mathcal{M}$ such that $\int \varphi d \nu=1$. Thus, (b) is satisfied by the definition of $\mathbf{F}$. To verify (a), simply note that

$$
\bmod _{p} \mathbf{E} \leq \bmod _{p} \mathbf{E} \cup \mathbf{F} \leq \int \varphi^{p} d m=\bmod _{p} \mathbf{E},
$$

since $\varphi$ is admissible for $\mathbf{E} \cup \mathbf{F}$ and $\varphi$ is extremal for the $p$-modulus of $\mathbf{E}$. It remains to establish (c). Assume that $f \in L^{p}(m)$ takes values in $[-\infty, \infty]$ and $\int f d \nu \geq 0$ for every $\nu \in \mathbf{F}$. Then for all $\varepsilon>0$ the metric $\varphi_{\varepsilon}=(\varphi+\varepsilon f)^{+} \geq 0$ belongs to $L^{p}(m)$ and $\int \varphi_{\varepsilon} d \nu \geq \int(\varphi+\varepsilon f) d \nu \geq \int \varphi d \nu=1$ for every $\nu \in \mathbf{F}$. If $\mu \in \mathbf{E}_{0}$, then there exists $0<c \leq 1$ such that $c \mu \in \mathbf{F}$ so that $\int \varphi_{\varepsilon} d \mu \geq c \int \varphi_{\varepsilon} d \mu=\int \varphi_{\varepsilon} d(c \mu) \geq 1$. Hence the metric $\varphi_{\varepsilon}$ is also admissible for $\mathbf{E}_{0}$. Thus,

$$
\int \varphi^{p} d m=\bmod _{p} \mathbf{E}_{0} \leq \int \varphi_{\varepsilon}^{p} d m=\int\left[(\varphi+\varepsilon f)^{+}\right]^{p} d m
$$

Then, Lemma 1 gives $\int \varphi^{p} d m \leq \int_{P_{\varepsilon}}\left[\varphi^{p}+p \varepsilon f \varphi^{p-1}\right] d m+o(\varepsilon) \cdot \varepsilon$, where the set $P_{\varepsilon}=\{\varphi+\varepsilon f>0\}$ and $o(\varepsilon) \rightarrow 0$ as $\varepsilon \rightarrow 0$. It follows that

$$
p \varepsilon \int_{P_{\varepsilon}} f \varphi^{p-1} d m \geq \int_{X \backslash P_{\varepsilon}} \varphi^{p} d m-o(\varepsilon) \cdot \varepsilon \geq-o(\varepsilon) \cdot \varepsilon .
$$

Dividing through by $p \varepsilon$ and letting $\varepsilon \rightarrow 0+$, we obtain

$$
\int f \varphi^{p-1} d m=\lim _{\varepsilon \rightarrow 0+} \int_{P_{\varepsilon}} f \varphi^{p-1} d m \geq 0,
$$

by the dominated convergence theorem. Therefore, condition $\left(B_{p}\right)$ holds if $\varphi$ is extremal for the $p$-modulus of $\mathbf{E}$. 


\section{Proof of Theorem 2 (Extremal metrics in $L^{1}$ )}

Let $\mathbf{E}$ be a measure system and let $\varphi$ be an admissible metric for $\mathbf{E}$ such that $\varphi \in L^{1}(m)$. If $\varphi=0 m$-a.e., then $\varphi$ is extremal for the 1-modulus of $\mathbf{E}$ and condition $\left(B_{1}\right)$ holds with $\mathbf{F}=\emptyset$. Thus, we assume that $0<\int \varphi d m<\infty$.

Suppose that condition $\left(B_{1}\right)$ holds. Let $\psi$ be an admissible metric for $\mathbf{E} \cup \mathbf{F}$ with $\psi \in L^{1}(m)$. Then $\int \psi d \nu \geq 1=\int \varphi d \nu$ for every $\nu \in \mathbf{F}$, by (b). Hence the function $f=\psi-\varphi \in L^{1}(m)$ takes values in $[-\infty, \infty], \int f d \nu \geq 0$ for all $\nu \in \mathbf{F}$ and $f(x) \geq 0$ whenever $\varphi(x)=0$. Since $f$ satisfies the hypothesis of (c), we obtain $\int(\psi-\varphi) d m \geq 0$. That is, $\int \varphi d m \leq \int \psi d m$, for every admissible metric $\psi$. Thus, $\varphi$ is extremal for the 1-modulus of $\mathbf{E} \cup \mathbf{F}$. It follows that $\bmod _{1} \mathbf{E} \leq \int \varphi d m=\bmod _{1} \mathbf{E} \cup \mathbf{F}=\bmod _{1} \mathbf{E}$, by (a). Therefore, $\varphi$ is extremal for the 1-modulus of $\mathbf{E}$.

Conversely, suppose that $\varphi$ is extremal for the 1-modulus of $\mathbf{E}$. Then $\varphi$ is also extremal for the 1-modulus of $\mathbf{E}_{0}=\left\{\mu \in \mathbf{E}: 1 \leq \int \varphi d \mu<\infty\right\}$. We want to check that condition $\left(B_{1}\right)$ holds. Assign $\mathbf{F}$ to be the family of all measures $\nu$ defined on $\mathcal{M}$ such that $\int \varphi d \nu=1$. Then (b) is satisfied automatically. For (a), $\bmod _{1} \mathbf{E} \leq$ $\bmod _{1} \mathbf{E} \cup \mathbf{F} \leq \int \varphi d m=\bmod _{1} \mathbf{E}$, since $\varphi$ is admissible for $\mathbf{E} \cup \mathbf{F}$ and $\varphi$ is extremal for the 1-modulus of $\mathbf{E}$. It remains to verify (c). Assume that $f \in L^{1}(m)$ takes values in $[-\infty, \infty], \int f d \nu \geq 0$ for all $\nu \in \mathbf{F}$ and $f(x) \geq 0$ whenever $\varphi(x)=0$. For all $\varepsilon>0$ the metric $\varphi_{\varepsilon}=(\varphi+\varepsilon f)^{+} \geq 0$ belongs to $L^{1}(m)$, and moreover, satisfies $\int \varphi_{\varepsilon} d \nu \geq \int(\varphi+\varepsilon f) d \nu \geq \int \varphi d \nu=1$ for every $\nu \in \mathbf{F}$. Now, for all $\mu \in \mathbf{E}_{0}$, there exists $0<c \leq 1$ such that $c \mu \in \mathbf{F}$. Thus, $\int \varphi_{\varepsilon} d \mu \geq c \int \varphi_{\varepsilon} d \mu=\int \varphi_{\varepsilon} d(c \mu) \geq 1$ for all $\mu \in \mathbf{E}_{0}$. This shows that the metric $\varphi_{\varepsilon}$ is also admissible for $\mathbf{E}_{0}$, and hence,

$$
\int \varphi d m=\bmod _{1} \mathbf{E}_{0} \leq \int \varphi_{\varepsilon} d m=\int(\varphi+\varepsilon f)^{+} d m=\int_{P_{\varepsilon}}(\varphi+\varepsilon f) d m,
$$

where $P_{\varepsilon}=\{\varphi+\varepsilon f>0\}$. This yields $\int_{P_{\varepsilon}} f d m \geq \varepsilon^{-1} \int_{X \backslash P_{\varepsilon}} \varphi d m \geq 0$ for all $\varepsilon>0$. As $\varepsilon \rightarrow 0+$, the characteristic functions $\chi_{P_{\varepsilon}}$ converge $m$-a.e. to the function $\chi_{P}$ where $P=\{\varphi>0\} \cup\{\varphi=0, f>0\}$ (convergence at $x \in X$ fails if $f(x)=-\infty$ ). Therefore, $\int_{P} f d m \geq 0$, and because we assumed that $f(x) \geq 0$ whenever $\varphi(x)=0$, we obtain $\int f d m=\int_{P} f d m+\int_{\{\varphi=0, f=0\}} f d m \geq 0$, as well. This completes the proof that condition $\left(B_{1}\right)$ holds whenever $\varphi$ is extremal for the 1-modulus of $\mathbf{E}$.

\section{Modification for curve families in $\mathbf{R}^{n}$}

The conditions $\left(B_{p}^{\prime}\right)$ of Corollaries 1 and 2 are sufficient tests for metrics to be extremal for $\bmod _{p} \Gamma$ by Theorems 1 and 2 . To verify that the conditions $\left(B_{p}^{\prime}\right)$ are also necessary, the proofs of Theorems 1 and 2 can be modified, as follows.

Let $1 \leq p<\infty$. Let $\Gamma$ be a curve family in $\mathbf{R}^{n}$ and let $\varphi$ be an extremal metric for the $p$-modulus of $\Gamma$ such that $0<\int_{\mathbf{R}^{n}} \varphi^{p}<\infty$. Then the metric $\varphi$ is also extremal for the $p$-modulus of $\Gamma_{0}=\left\{\gamma \in \Gamma: 1 \leq \int_{\gamma} \varphi d s<\infty\right\}$. We want to check that condition $\left(B_{p}^{\prime}\right)$ holds. Assign $\Gamma^{\prime}$ to be the family of all curves $\gamma$ in $\mathbf{R}^{n}$ such that $\int_{\gamma} \varphi d s=1$. Then (b) holds by definition. To show (a), simply note that $\bmod _{p} \Gamma \leq \bmod _{p} \Gamma \cup \Gamma^{\prime} \leq \int_{\mathbf{R}^{n}} \varphi^{p}=\bmod _{p} \Gamma$, because $\varphi$ is admissible for $\Gamma \cup \Gamma^{\prime}$ and $\varphi$ is extremal for the $p$-modulus of $\Gamma$. It remains to verify (c). Assume that $f \in L^{p}\left(\mathbf{R}^{n}\right)$ takes values in $[-\infty, \infty]$ and $\int_{\gamma} f d s \geq 0$ for all $\gamma \in \Gamma^{\prime}$. In the case $p=1$, also assume that $f(x) \geq 0$ whenever $\varphi(x)=0$. For all $\varepsilon>0$, the metric $\varphi_{\varepsilon}=(\varphi+\varepsilon f)^{+} \geq 0$ belongs to $L^{p}\left(\mathbf{R}^{n}\right)$. Moreover, $\int_{\gamma} \varphi_{\varepsilon} d s \geq \int_{\gamma}(\varphi+\varepsilon f) d s \geq \int_{\gamma} \varphi d s=1$ for all $\gamma \in \Gamma^{\prime}$. 
If $\gamma \in \Gamma_{0}$, then

$$
1 \leq \int_{\gamma} \varphi d s=\sum_{i} \int_{a_{i}}^{b_{i}} \varphi\left(\gamma_{i}(t)\right)\left|\gamma_{i}^{\prime}(t)\right| d t<\infty .
$$

Since each term in the line integral is non-negative and finite, the function

$$
c \mapsto \int_{a_{i}}^{c} \varphi\left(\gamma_{i}(t)\right)\left|\gamma_{i}^{\prime}(t)\right| d t
$$

on $\left[a_{i}, b_{i}\right]$ is continuous for each $i$. Hence we can pick $c_{i} \in\left[a_{i}, b_{i}\right]$ for all $i$ in such a way that the subcurve $\gamma_{1}=\bigsqcup_{i} \gamma\left(\left[a_{i}, c_{i}\right]\right)$ of $\gamma$ satisfies $\int_{\gamma_{1}} \varphi d s=1$. This means that $\gamma_{1} \in \Gamma^{\prime}$. Thus, $\int_{\gamma} \varphi_{\varepsilon} d s \geq \int_{\gamma_{1}} \varphi_{\varepsilon} d s \geq 1$. This shows that $\varphi_{\varepsilon}$ is also admissible for $\Gamma_{0}$. Hence

$$
\int_{\mathbf{R}^{n}} \varphi^{p}=\bmod _{p} \Gamma_{0} \leq \int_{\mathbf{R}^{n}} \varphi_{\varepsilon}^{p}=\int_{\mathbf{R}^{n}}\left[(\varphi+\varepsilon f)^{+}\right]^{p} .
$$

To finish checking (c), one can now proceed as above. Follow the argument from section 3 , when $1<p<\infty$, and follow the argument from section 4 , when $p=1$.

\section{Proof of Theorem 4}

Suppose that $\varphi: \mathbf{R}^{n} \rightarrow[0, \infty]$ is a Borel function and let $\Gamma_{\varphi}$ be the family of all curves $\gamma$ in $\mathbf{R}^{n}$ such that $\int_{\gamma} \varphi d s \geq 1$. Fix any $1 \leq p<\infty$ such that $0<\int_{\mathbf{R}^{n}} \varphi^{p}<\infty$. We want to show that $\varphi$ is extremal for the $p$-modulus of $\Gamma_{\varphi}$. For each $y \in \mathbf{R}^{n}$ let $\ell_{y}=y+\mathbf{R} e_{1} \cong \mathbf{R}$ denote the line through $y$ parallel to the direction $e_{1}=(1,0, \ldots, 0)$. By Fubini's theorem, $\varphi \in L^{p}\left(\ell_{y}\right), y=(0, \bar{y})$ for $H^{n-1}$-a.e. $\bar{y} \in \mathbf{R}^{n-1}$. In particular, we also have $\varphi \in L_{\text {loc }}^{1}\left(\ell_{y}\right), y=(0, \bar{y})$ for $H^{n-1}$-a.e. $\bar{y} \in \mathbf{R}^{n-1}$. Here, as above and as below, $H^{s}$ denotes $s$-dimensional Hausdorff measure. Below $|I|$ denotes the diameter of an interval $I$.

Lemma 2. Suppose that $\varphi \in L_{\mathrm{loc}}^{1}\left(\ell_{y}\right)$. Then, for $H^{1}$-a.e. $x \in \ell_{y}$ such that $\varphi(x)>0$, there exist a sequence of positive integers $n_{k}=n_{k}(x) \rightarrow \infty$ and a sequence intervals $I_{k}=I_{k}(x) \subset \ell_{y}$ centered at $x$ with $\left|I_{k}\right| \rightarrow 0$ such that $\int_{I_{k}} \varphi d t=1 / n_{k}$ for all $k$.

Proof. Define the function $g_{x}(r)=\int_{-r}^{r} \varphi\left(x+t e_{1}\right) d t$ for all $x \in \ell_{y}$ and $r \geq 0$. Then $\lim _{r \rightarrow 0+} g_{x}(r) / 2 r=\varphi(x)$ for $H^{1}$-a.e. $x \in \ell_{y}$, by the Lebesgue differentiation theorem. Hence for $H^{1}$-a.e. $x \in \ell_{y}$ such that $\varphi(x)>0$, there exists $r_{0}=r_{0}(x)>0$ such that $0<g_{x}(r)<\infty$ for all $0<r \leq r_{0}$. Since $\left.g_{x}\right|_{\left[0, r_{0}\right]}$ is continuous and $g_{x}(0)=0$, we can find a sequence of integers $n_{k}=n_{k}(x)$ and a sequence of radii $r_{k}=r_{k}(x) \rightarrow 0$ such that $g_{x}\left(r_{k}\right)=1 / n_{k}$. Then $I_{k}=I_{k}(x)=x+\left[-r_{k}, r_{k}\right] e_{1} \subset \ell_{y}$ is a sequence of intervals with the desired property.

Let $E \subset \mathbf{R}^{n}$ be the set of points $x \in \mathbf{R}^{n}$ where the conclusion of Lemma 2 holds, i.e. $x \in E$ if and only if there exist a sequence of positive integers $n_{k}=n_{k}(x) \rightarrow \infty$ and a sequence of intervals $I_{k}=I_{k}(x) \subset \ell_{x}$ centered at $x$ with $\left|I_{k}\right| \rightarrow 0$ such that $\int_{I_{k}} \varphi d t=1 / n_{k}$. By Fubini's theorem and Lemma 2, we have $x \in E$ for a.e. $x \in \mathbf{R}^{n}$ such that $\varphi(x)>0$. We define a curve family $\Gamma^{\prime} \subset \Gamma_{\varphi}$ as follows. Choose one pair of sequences $\left(n_{k}(x)\right)_{k=1}^{\infty}$ and $\left(I_{k}(x)\right)_{k=1}^{\infty}$ for each $x \in E$. Then, for each $x \in E$ and $k \geq 1$, define a curve $\gamma_{k}(x)=\bigsqcup_{i=1}^{n_{k}} I_{k}(x)$, i.e. let $\gamma_{k}(x)$ be a curve which covers the interval $I_{k}(x)$ exactly $n_{k}(x)$-times. Set $\Gamma^{\prime}=\left\{\gamma_{k}(x): x \in E\right.$ and $\left.k \geq 1\right\}$. 
To prove that $\varphi$ is extremal for the $p$-modulus of $\Gamma_{\varphi}$, it is enough by either Corollary 1 or Corollary 2 (according to whether $1<p<\infty$ or $p=1$ ) to show that $\left(B_{p}^{\prime}\right)$ holds for $\Gamma^{\prime}$. To start note $\int_{\gamma_{k}(x)} \varphi d s=n_{k}(x) \int_{I_{k}(x)} \varphi d t=n_{k}(x) / n_{k}(x)=1$ for all $\gamma_{k}(x) \in \Gamma^{\prime}$. This shows that (b) holds. And, since $\Gamma^{\prime} \subset \Gamma_{\varphi}$, (a) is true too. To verify (c), assume that $f \in L^{p}\left(\mathbf{R}^{n}\right)$ takes values in $[-\infty, \infty]$ and $\int_{\gamma_{k}(x)} f d s \geq 0$ for all $\gamma_{k}(x) \in \Gamma^{\prime}$. Moreover, if $p=1$, assume that $f(x) \geq 0$ whenever $\varphi(x)=0$. By Fubini's theorem and the Lebesgue differentiation theorem,

$$
f(x)=\lim _{k \rightarrow \infty} \frac{1}{\left|I_{k}(x)\right|} \int_{I_{k}(x)} f d t
$$

for a.e. $x \in E$, and in particular, for a.e. $x \in \mathbf{R}^{n}$ such that $\varphi(x)>0$. By assumption,

$$
\int_{I_{k}(x)} f d t=\frac{1}{n_{k}(x)} \int_{\gamma_{k}(x)} f d s \geq 0 \quad \text { for all } x \in E \text { and } k \geq 1 .
$$

Thus, combining the two displayed equations, $f(x) \geq 0$ at a.e. $x \in \mathbf{R}^{n}$ such that $\varphi(x)>0$. It follows that $\int_{\mathbf{R}^{n}} f \varphi^{p-1} \geq 0$, if $1<p<\infty$, and $\int_{\mathbf{R}^{n}} f \geq 0$, if $p=1$. Hence (c) holds. Therefore, $\left(B_{p}^{\prime}\right)$ holds and $\varphi$ is extremal for the $p$-modulus of $\Gamma_{\varphi}$.

\section{References}

[1] Ahlfors, L.: Conformal invariants: Topics in geometric function theory. - McGraw-Hill Book Co., New York, 1973.

[2] Ahlfors, L., and A. Beurling: Conformal invariants and function-theoretic null-sets. - Acta Math. 83, 1950, 101-129.

[3] Bishop, C. J., and H. Hakobyan: Frequency of dimension distortion under quasisymmetric mappings. - Preprint, arXiv:1211.0233.

[4] Bonk, M.: Uniformization of Sierpiński carpets in the plane. - Invent. Math. 186:3, 2011, 559-665.

[5] Evans, L. C., and R. F. Gariepy: Measure theory and fine properties of functions. - Stud. Adv. Math., CRC Press, Boca Raton, FL, 1992.

[6] Fuglede, B.: Extremal length and functional completion. - Acta Math. 98, 1957, 171-219.

[7] Garnett, J. B., and D. E. Marshall: Harmonic measure. - New Math. Monogr. 2, Cambridge Univ. Press, Cambridge, 2005.

[8] Kahn, J., and M. Lyubich: Local connectivity of Julia sets for unicritical polynomials. - Ann. of Math. (2) 170:1, 2009, 413-426.

[9] Hakobyan, H.: Conformal dimension: Cantor sets and Fugledge modulus. - Int. Math. Res. Not. 2010:1, 2010, 87-111.

[10] Heinonen, J.: Lectures on analysis on metric spaces. - Universitext, Springer-Verlag, New York, 2001.

[11] Heinonen, J.: Nonsmooth calculus. - Bull. Amer. Math. Soc. (N.S.) 44:2, 2007, 163-232.

[12] Jenkins, J.: The method of the extremal metric. - In: The Bieberbach Conjecture (West Lafayette, Ind., 1985), Math. Surveys Monogr. 21, Amer. Math. Soc., Providence, RI, 1986, 95-104.

[13] Milnor, J.: Local connectivity of Julia sets: expository lectures. - In: The Mandelbrot set, theme and variations (Lei Tan, ed.), London Math. Soc. Lecture Note Ser. 274, Cambridge Univ. Press, Cambridge, 2000, 67-116.

[14] Ohtsuka, M.: Dirichlet problem, extremal length and prime ends. - Van Nostrand Reinhold Company, New York, 1970. 
[15] Rodin, B.: The method of extremal length. - Bull. Amer. Math. Soc. 80, 1974, 587-606.

[16] Schramm, O.: Transboundary extremal length. - J. Anal. Math. 66, 1995, 307-329.

[17] VÄIsÄLÄ, J.: Lectures on $n$-dimensional quasiconformal mappings. - Lecture Notes in Math. 229, Springer-Verlag, Berlin, 1971.

[18] Vuorinen, M.: Conformal geometry and quasiregular mappings. - Lecture Notes in Math. 1319, Springer-Verlag, Berlin, 1988.

[19] Wolf, M., and B. Zwiebach: The plumbing of minimal area surfaces. - J. Geom. Phys. 15, 1994, 23-56.

Received 31 August 2012 • Accepted 23 November 2012 